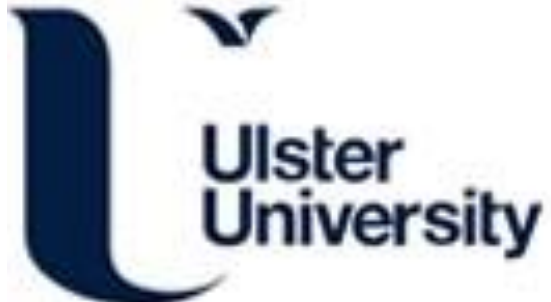

\section{Detection of Straight Lines Using a Spiking Neural Network Model}

Wu, Q., McGinnity, TM., Maguire, L., Valderrama, G., \& Cai, J. (2009). Detection of Straight Lines Using a Spiking Neural Network Model. In Unknown Host Publication (Vol. 2, pp. 385-389). IEEE Xplore. https://doi.org/10.1109/ICNC.2009.484

Link to publication record in Ulster University Research Portal

\section{Published in:}

Unknown Host Publication

Publication Status:

Published (in print/issue): 28/12/2009

DOI:

10.1109/ICNC.2009.484

\section{Document Version}

Publisher's PDF, also known as Version of record

\section{General rights}

Copyright for the publications made accessible via Ulster University's Research Portal is retained by the author(s) and / or other copyright owners and it is a condition of accessing these publications that users recognise and abide by the legal requirements associated with these rights.

\section{Take down policy}

The Research Portal is Ulster University's institutional repository that provides access to Ulster's research outputs. Every effort has been made to ensure that content in the Research Portal does not infringe any person's rights, or applicable UK laws. If you discover content in the Research Portal that you believe breaches copyright or violates any law, please contact pure-support@ulster.ac.uk. 


\section{Detection of Straight Lines Using a Spiking Neural Network Model}

\author{
QingXiang Wu, T.M. McGinnity, Liam Maguire, \\ G.D. Valderrama-Gonzalez \\ Intelligent Systems Research Centre \\ University of Ulster at Magee \\ Londonderry, BT48 7JL, N. Ireland, UK \\ \{q.wu, tm.mcginnity, lp.maguire, \\ g.valderrama\}@ulster.ac.uk
}

\author{
Jianyong Cai \\ School of Physics and OptoElectronic Technology \\ Fujian Normal University \\ Fujian, Fuzhou, 350007, China \\ \{qxwu, cjy\}@fjnu.edu.cn
}

\begin{abstract}
Receptive fields of neurons play various roles in biological neural networks. Based on a receptive field with the function of Hough transform, a spiking neural network model is proposed to detect straight lines in a visual image. Through the network, straight lines transform to corresponding neurons with high firing rates in the output neuron array. Simulation results show that straight lines can be detected by the network and firing rates of the corresponding neurons are referred to lengths of the lines. This model can be used to explain how a spiking neuron-based network can detect straight lines, and furthermore the model can be used in an artificial intelligent system.
\end{abstract}

Keywords-spiking neural networks; straght line detection; Hough transform; receptive field

\section{INTRODUCTION}

Receptive fields of neurons can be found at different positions in the visual system and play different roles. For example, different receptive fields have been studied in [1, 2]. Biological evidence shows that receptive fields of sensory cortical neurons have some plasticity, changing in response to alterations of neural activity or sensory experience [3-5]. In order to extract features from images of an environment promptly, the visual system must be capable of adapting rapidly to changing scenes. Individual neurons in the visual cortex respond to light stimuli in receptive fields on the retina, and the structure of these receptive fields can change in different contexts [6]. The results in [6] indicate that temporal and spatial restructuring of visual cortical receptive fields may be important in controlling the resolution of visual processing in the primary visual cortex in a statedependent way. By comparing the structure of receptive fields recorded from the different layers of the cat's primary visual cortex, the receptive fields vary with layer in the primary visual cortex [7]. In order to read out, or decode, mental content from brain activity, a decoding method based on quantitative receptive-field models is proposed to characterize the relationship between visual stimuli and neuron activity in early visual areas [8].

A challenging goal is to apply all biological findings to artificial intelligent systems to make human-like intelligent systems. As whole neuronal circuits in the brain are too complicated to be drawn using anatomical technology, most biological findings are only refer to single neurons and local circuits in the biological nerve system. The receptive fields in the visual system can be developed by training [9-11]. During development of the visual system, the pattern of visual inputs may have an instructive role in refining developing neural circuits. How visual inputs of specific spatiotemporal patterns shape the circuit development remains largely unknown [10]. Therefore, in order to apply the findings to solve problems in artificial intelligent systems, it is required to create a structure based on the properties of single neurons and local neuronal circuits, or modeling of the biological system in a way based on electronic circuits. For example, a lot of researchers have studied simulations of the human visual system, which is called artificial vision system [12]. Based on the receptive fields inspired by biological system, different spiking neural network models were used to edge and motion detections $[13,14]$ and feature extraction [15]. The visual system, in which spiking neurons are basic units for information processing, has powerful intelligent functions to process visual images. It can be boldly predicted that most image processing functions can be implemented using different spiking neuron-based networks. In this paper, a spiking neural network model is proposed to detect straight lines in a visual image. Simulation results show that the straight-line detection can be completed within 100ms time if the model is implemented in fully parallel by electronic units whose processing speed is the same as biological neurons.

The remainder of this paper is organized as follows. In Section 2, the architecture of the network and receptive fields of neurons are detailed. The simulation algorithm is described in Section 3. The simulation results are shown in Section 4. Discussions are presented in Section 5.

\section{ARCHITECTURE OF SPIKING NEURAL NETWORK FOR DETECTION OF STRAIGHT LINES}

The brain can memorize features of various objects and then recognize the seen objects promptly. However, it is still an open question for what is exact architecture of neural network that represents the seen objects in the brain. For simplicity, an input image in our proposed model is represented by a neuron array in the $\mathrm{x}-\mathrm{y}$ plan, as shown in Fig. 1. The input image usually is the outputs of the edge detection neural network [13]. Straight lines on the plan can 
be identified by a pair of parameters $(\rho, \theta)$. $\rho$ is the distance from the line to original point $(0,0), \theta$ is the angle related to the line as shown in Fig.1. For example, we have $\rho_{1}=\sqrt{50}$ and $\theta_{1}=45^{\circ}$ for line $l_{1}, \rho_{2}=4$ and $\theta_{2}=0^{\circ}$ for line $l_{2}$.

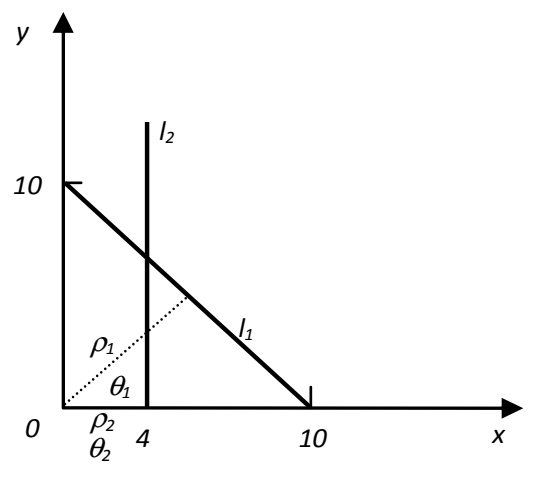

Figure 1. Straight lines in an image.

Suppose that $\rho$ and $\theta$ are represented by a neuron array. Therefore, each neuron in the array corresponds to a straight line in the $x-y$ plan. If we set the receptive field of each neuron in the $\rho-\theta$ array to the corresponding line, a spiking neural network model is presented in Fig. 2. For example, neuron $\mathrm{N} 1$ at $\left(\rho=7, \theta=45^{\circ}\right)$ has excitatory synapses from a receptive field of line $l_{l}$ while neuron N2 at $\left(\rho=4, \theta=0^{\circ}\right)$ has excitatory synapses from a receptive field of line $l_{2}$. Therefore, neuron $\mathrm{N} 1$ responds to line $l_{l}$ with high firing rate while neuron $\mathrm{N} 2$ responds to line $l_{2}$ with high firing rate.

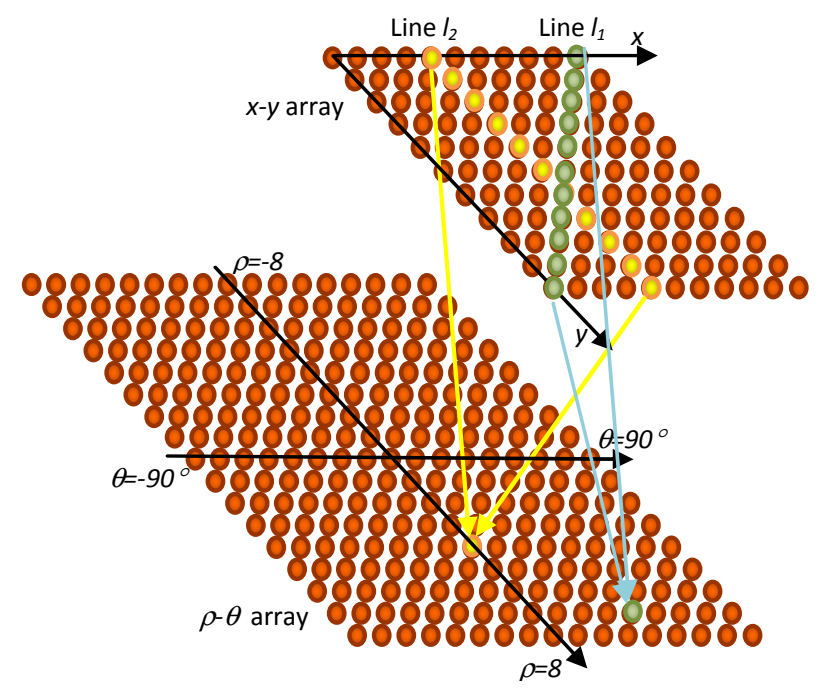

Figure 2. Architecture of Spiking Neural Network Model

\section{ALGORITHM FOR SIMULATION OF THE NETWORK}

The Simulation results show that the conductance based integrate-and-fire model is very close to the Hodgkin and Huxley neuron model [11]. The conductance based integrate-and-fire model is applied to the aforementioned network model. Let $S_{x, y}(\mathrm{t})$ represent spike train from $x-y$ array.

$S_{x, y}(t)= \begin{cases}1 & \text { if neuron }(x, y) \text { fires at time } t . \\ 0 & \text { if neuron }(x, y) \text { does not fire at time } t .\end{cases}$

Let $g_{e x(x, y)}(t)$ represent the dynamic conductance for excitatory synapses. According to the conductance based integrate-and-fire neuron model [11-17], the conductance of the excitatory synapse is governed by

$$
\frac{d g_{e x(x, y)}(t)}{d t}=-\frac{1}{\tau_{e x}} g_{e x(x, y)}(t)+q S_{x, y}(t),
$$

where $q$ is a peak conductance of a synapse, $\tau_{e x}$ is the time constant of excitatory synapses. Neuron $\left(\rho_{n}, \theta_{m}\right)$ at visual image neuron array is governed by

$c_{m} \frac{d v_{\rho_{n}, \theta_{m}}(t)}{d t}=g_{l}\left(E_{l}-v_{\rho_{n}, \theta_{m}}(t)\right)+$

$\sum_{(x, y) \in R F_{\rho_{n}, \theta_{m}}} \frac{w_{e x(x, y) \rightarrow\left(\rho_{n}, \theta_{m}\right)} g_{e x(x, y)}(t)}{A_{e x}}\left(E_{e x}-v_{\rho_{n}, \theta_{m}}(t)\right)$,

where $v_{\rho_{n}, \theta_{m}}(t)$ is the membrane potential of neuron $\left(\rho_{n}, \theta_{m}\right)$, $E_{e x}$ is the reverse potential for excitatory synapses, $c_{m}$ represents a capacitance of the membrane, $g_{l}$ represents the conductance of membrane, ex is short for excitatory, $A_{e x}$ is the membrane surface area connected to a excitatory synapse, $R F_{\rho_{n}, \theta_{m}}$ represents a receptive field corresponding to a line on the $x-y$ plan, $w_{e x(x, y) \rightarrow\left(\rho_{n}, \theta_{m}\right)}$ is a connection weight from neuron $(x, y)$ to neuron $\left(\rho_{n}, \theta_{m}\right)$. The neuron model is simulated by the Euler method using a time step of $0.1 \mathrm{~ms}$. When the potential $v_{\rho_{n}, \theta_{m}}(t)$ is larger than threshold $v_{t h}$, the neuron generates a spike and proceeds to refractory period for time $\tau_{r e f}$. After the refractory period the neuron will start to integrate inputs from a potential $v_{\text {rest }}$. $w_{e x(x, y) \rightarrow\left(\rho_{n}, \theta_{m}\right)}$ can be set according to Hough Transform $[18,19]$ as follows.

$$
w_{e x(x, y) \rightarrow\left(\rho_{n}, \theta_{m}\right)}=\left\{\begin{array}{l}
1 \text { if } \rho_{n}=x \cos \left(\theta_{m}\right)+y \sin \left(\theta_{m}\right) . \\
0 \text { if } \rho_{n} \neq x \cos \left(\theta_{m}\right)+y \sin \left(\theta_{m}\right) .
\end{array}\right.
$$

Neuron $\left(\rho_{n}, \theta_{m}\right)$ generates a spike train that is represented by

$$
S_{\rho_{n}, \theta_{m}}(t)=\left\{\begin{array}{l}
1 \text { if neuron }\left(\rho_{n}, \theta_{m}\right) \text { fires at time } t . \\
0 \text { if neuron }\left(\rho_{n}, \theta_{m}\right) \text { doesn't fire at time } t .
\end{array}\right.
$$

The firing rate of the output neuron array can be calculated by 


$$
r_{\rho_{n}, \theta_{m}}(t+T)=\frac{1}{T} \sum_{t}^{t+T} S_{\rho_{n}, \theta_{m}}(t) .
$$

The peaks of firing rates in the output array correspond to the straight lines in the $x-y$ array. However, based on this algorithm, a large weight array $w_{e x(x, y) \rightarrow\left(\rho_{n}, \theta_{m}\right)}$ will be encountered. For example, suppose that dimension $x_{\max } \times y_{\max }$ of $x-y$ array is $500 \times 500$. The largest $\rho$ is as follows.

$$
\rho_{\max }=\sqrt{x_{\max }^{2}+y_{\max }^{2}}=\sqrt{500^{2}+500^{2}}=707 .
$$

Resolution of $\theta$ is set to $1^{\circ}$. The dimension of the $\rho-\theta$ array is $180 \times 1414$. The dimension of $w_{\operatorname{ex}(x, y) \rightarrow\left(\rho_{n}, \theta_{m}\right)}$ is $500 \times 500 \times 180 \times 1414$. In order to avoid the large number of connections, delivery fields of neuron $(x, y)$ is defined as a set of neurons whose labels $\left(\rho_{n}, \theta_{m}\right)$ are satisfied $\rho_{n}=x \cos \left(\theta_{m}\right)+y \sin \left(\theta_{m}\right)$. The spike trains are deliver to the neurons in the delivery fields instead of all neurons in the $\rho-\theta$ array. Therefore simulation is speeded up.

\section{RESUlTS OF THE SIMULATION}

The model has been simulated using Matlab program. In the simulation, the parameters are set as follows: $v_{t h}=-60$ mv. $v_{\text {reset }}=-70 \mathrm{mv} . E_{e x}=0 \mathrm{mv} . E_{i h}=-75 \mathrm{mv} . E_{l}=-70 \mathrm{mv} . g_{l}$ $=1.0 \mu \mathrm{s} / \mathrm{mm}^{2} . c_{m}=8 \mathrm{nF} / \mathrm{mm}^{2}$. $\tau_{e x}=4 \mathrm{~ms}$. $A_{e x}=0.03125 \mathrm{~mm}^{2}$. $g_{l}=1.0 \mathrm{us} / \mathrm{mm}^{2} . \mathrm{T}=100 \mathrm{~ms} . \quad q=0.0006 . \quad t_{\text {ref }}=3 \mathrm{~ms}$. These parameters can be adjusted to get good quality of output image. Each neuron corresponds to a pixel on the $x-y$ array. Each neuron on the line generates a Poisson spike train with mean frequency $60 \mathrm{~Hz}$, as shown in Fig. 3.

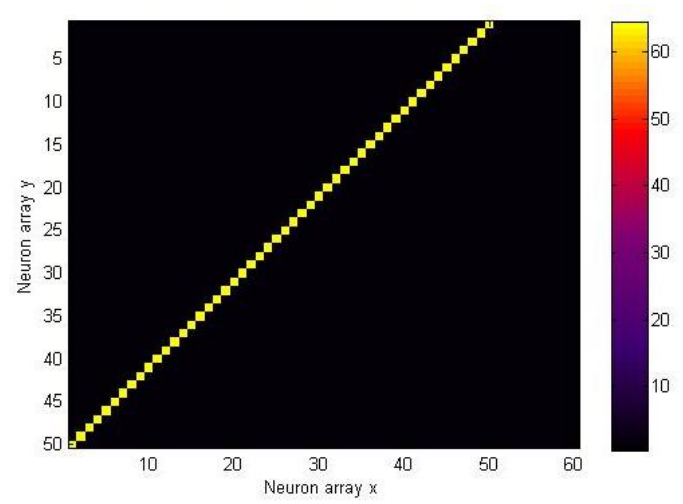

Fig.3. Firing rate map for the $x-y$ array.

If the line in Fig. 3 is presented to the network model, a firing rate map is obtained in the $\rho-\theta$ array as shown in Fig. 4(a) or (b). Firing rate map (a) is obtained using $q=0.01$, while firing rate map (b) is obtained using $q=0.0006$. It can be seen that a line in the $x-y$ array can be represented by one peak-firing-rate neuron in the $\rho-\theta$ array. Reducing parameter $q$, we can only see firing of the corresponding neuron as shown in Fig. 4(b).

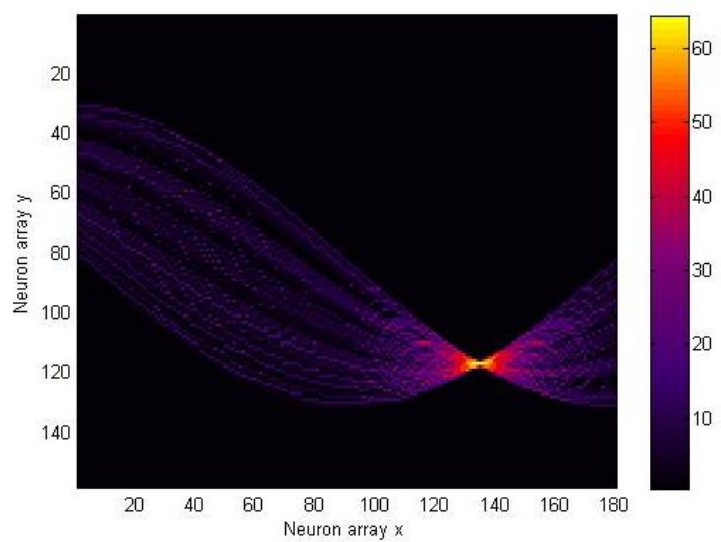

Figure 4 (a) Firing rate map with $q=0.01$

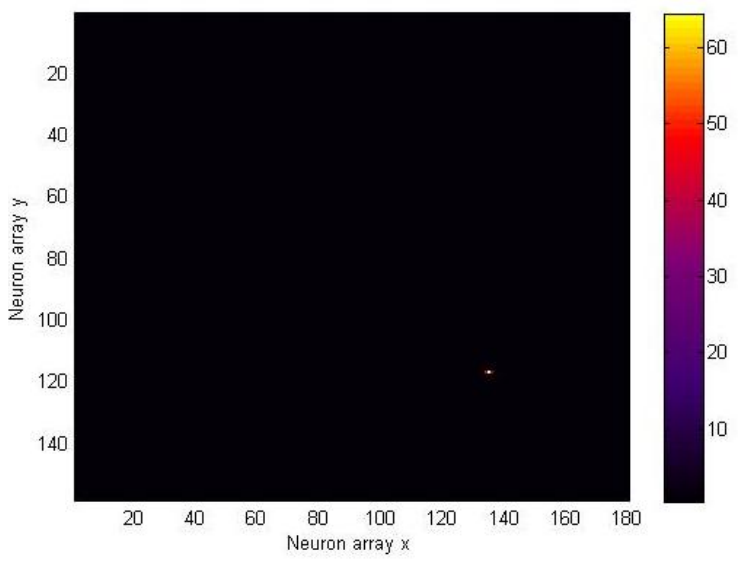

Fig.4 (b) Firing rate map with $q=0.0006$

In this case, the neuron with the peak firing rate is Neuron $(135,113)$. As $\rho_{\max }=\sqrt{50^{2}+60^{2}}=78$ is set according to the dimension of the $x-y$ array, the $\rho-\theta$ array should be set 156 rows and 180 columns corresponding to $\rho$ and $\theta$ respectively. Suppose that Row 78 corresponds to $\rho=0$. The rows less than 78 correspond to $-\rho$. Analogy, suppose that column 90 is set to correspond to $\theta=0^{\circ}$, and then the columns less than 90 correspond to $-\theta$. Therefore, Neuron $(135,113)$ correspond to $\rho=35$ and $\theta=45^{\circ}$. This is comparable with the Hough Transform shown in Fig. 5.

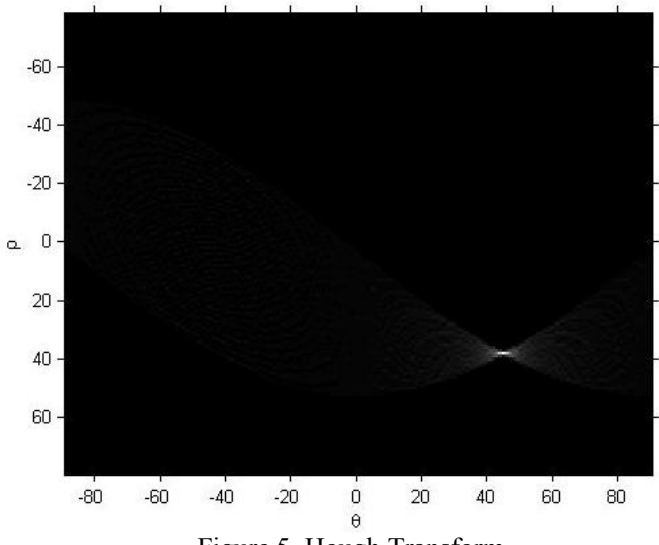

Figure 5. Hough Transform. 


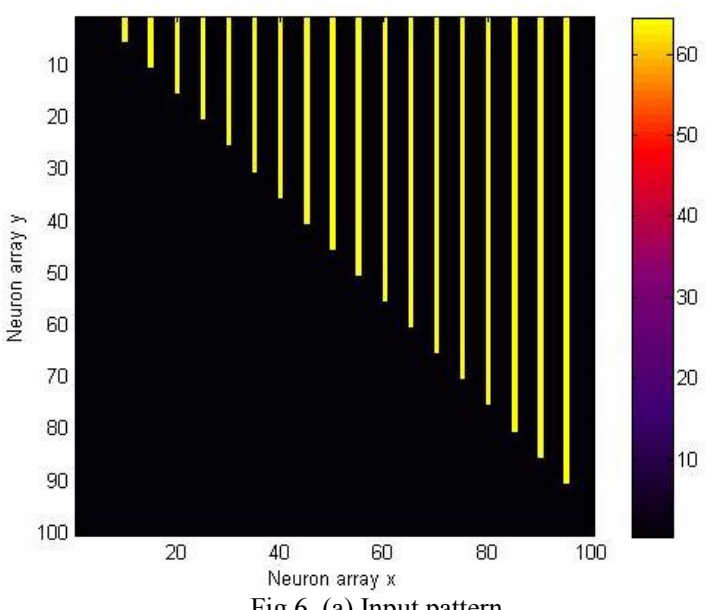

Fig.6. (a) Input pattern

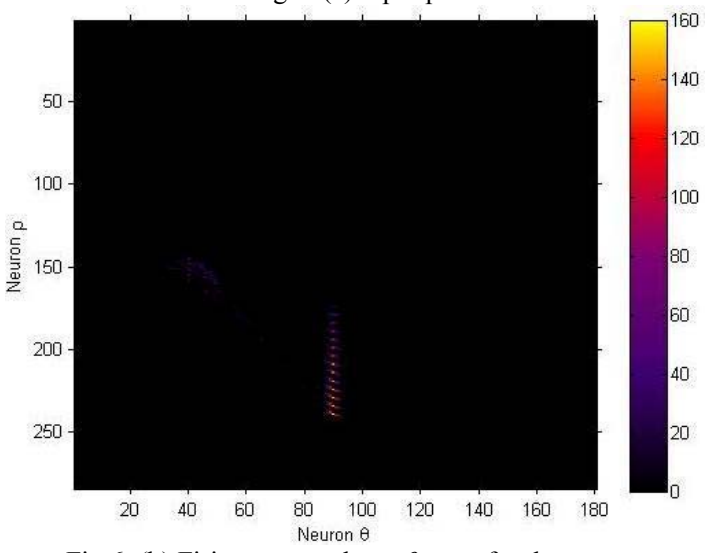

Fig.6. (b) Firing rate on the $\rho-\theta$ array for the pattern

In order to investigate the properties of this network, we have presented to the network a pattern with multiple lines with different length to the network as shown in Fig. 6(a). A firing rate map is obtained in Fig. 6(b). Peak-firing-rate neurons are obtained in the column 90 that corresponds to $\theta$ $=0^{\circ}$ for all lines. The largest peak-firing-rate neuron is Neuron $(90,237)$.

In this case, $\rho_{\max }=142$. Suppose that row 142 corresponds to $\rho=0$. Neuron $(90,237)$ corresponds to the line with $\rho=95$ and $\theta=0^{\circ}$. The least peak-firing-rate neuron is Neuron $(90,177)$ corresponding to the line with $\rho=35$ and $\theta=0^{\circ}$. There are no firing neurons corresponding to the lines at $\rho=30,25,20,15$, and 10 . This means that lines shorter than the line at $\rho=35$ cannot be detected by the network with the parameters. By adjusting the parameter $q$ or $v_{t h}$, we can determine how long a line can be detected by the network. In this case, a line longer than the line at $\rho=35$, which is 35 pixels, can be detected by the corresponding neuron with a peak-firing-rate about $20 \mathrm{~Hz}$. The longer the line is, the higher the corresponding neuron fires. Length of line at $\rho=95$ is 95 pixels. The firing rate of the corresponding neuron is about $160 \mathrm{~Hz}$. The firing rate vs length of a line is shown in Fig. 7. The value $q$ is determined according to how long of the line should be

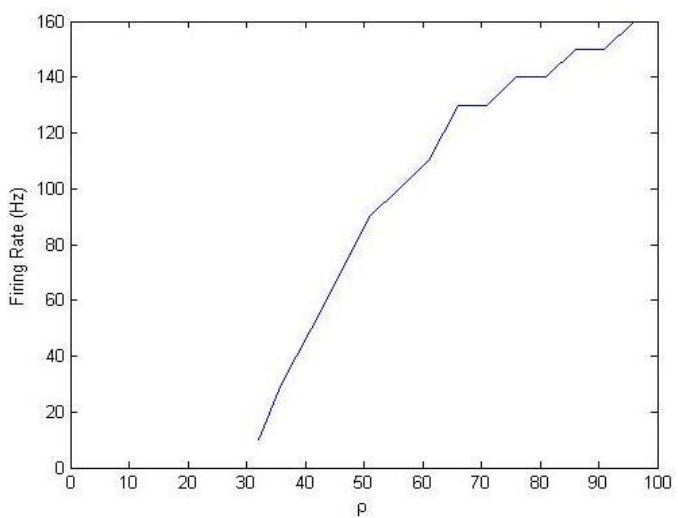

Figure 7. Peak firing rate vs length of lines.

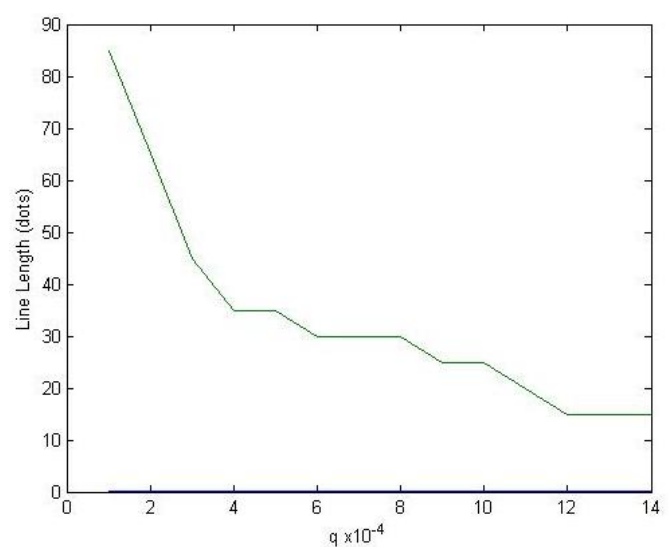

Figure 8. Detectable shortest lines vs values of $q$.

detected. The larger the value $q$ is, the shorter the detectable line is. The value $q$ vs the shortest detectable line is shown in Fig. 8.

\section{DISCUSSIONS}

This paper presents a spiking neural network model in which each neuron in the output array responds to a specific line in a visual image. The spiking rates of the neurons correspond to lengths of specific lines. By adjusting the parameters, the neurons can ignore the lines that are shorter than a specific value. Based on an implementation of large scale parallel spiking neural networks, the principle can be used in artificial intelligent systems. The principle can also be used to explain how a spiking neuron based network can perform the Hough-like transform and detects straight lines. However, there are still discussion questions that would be studied further. Firstly, how the model can exist in the biological visual system, for example, the visual cortex layers [7]. Secondly, it seems that the receptive field is possible to be developed based on the principle of spike timing dependent plasticity of synapses [9]. Finally, there are different receptive fields in the visual system and we need to investigate how the visual system can switch among 
the different receptive fields in response of different visual images.

\section{REFERENCES}

[1] E. R. Kandel, and H. J. Shwartz, Principles of neural science. Edward Amold (Publishers) Ltd., 1981.

[2] D. H. Hubel, and T.N. Wiesel, "Receptive field, binocular interaction and functional architecture in the cat's visual cortex", J. Physiol. vol. 160, pp.106-164, 1962.

[3] M. P. Kilgard, and M. M.Merzenich, "Cortical map reorganization enabled by nucleus basalis activity", Science, vol. 279, pp.1714$1718,1998$.

[4] F. J. Shamma, S. Elhilali, M. Klein, "Rapid task-related plasticity of spectrotemporal receptive fields in primary auditory cortex", Nature Neurosci. Vol. 6, pp.1216-1223, 2003.

[5] R. C. Froemke, M. M. Merzenich, and C. E. Schreiner, "A synaptic memory trace for cortical receptive field plasticity", Nature, vol. 450, pp. $425-429,2007$.

[6] F. Wargatter, K. Suder, Y. Zhao, N. Kerscher, U. T. Eysel, K. Funke, "State-dependent receptive-field restructuring in the visual cortex", Nature, vol. 396, pp.165 - 168, 1998.

[7] [7] L. M. Martinez, Q. Wang, R. C. Reid, C. Pillai, J. Alonso, F. T. Sommer, J. A Hirsch, "Receptive field structure varies with layer in the primary visual cortex", Nature Neuroscience, vol. 8, pp.372 $379,2005$.

[8] [8] K. N. Kay, T. Naselaris, R. J. Prenger, J. L. Gallant, Identifying natural images from human brain activity, Nature, vol. 452, pp.352 $355,2008$.

[9] Q.X. Wu, T.M. McGinnity, L. P. Maguire, A. Belatreche, and B.Glackin, " Simulation of Intelligent Computational Models in Biological Systems", Proc. of International Conference on Machine
Learning and Cybernetics (ICMLC), IEEE, New York, 4 pp.19741979, 2007.

[10] F. Engert, H. W. Tao, L. I. Zhang, M. Poo, "Moving visual stimuli rapidly induce direction sensitivity of developing tectal neurons", Nature, vol. 419, pp.470 - 475, 2002.

[11] Q.X. Wu, T.M. McGinnity, L. P. Maguire, A. Belatreche, and B. Glackin, "2D Co-ordinate Transformation Based on a Spike TimingDependent Plasticity Learning Mechanism", Journal of Neural Networks, vol.21, pp.1318-1327, 2008.

[12] A. Bharath and M. Petrou, Next generation artificial vision system, reverse engineering the human visual system, Artech House, 2008.

[13] Q.X. Wu, T.M. McGinnity, L. P. Maguire, A. Belatreche, and B.Glackin, "Edge Detection Based on Spiking Neural Network Model", Springer-Verlag Berlin Heidelberg, LNAI 4682 pp.26-34, 2007.

[14] Q.X. Wu, T.M. McGinnity, L. P. Maguire, J. Cai, G. Valderrama, "Motion Detection Using Spiking Neural Network Model", SpringerVerlag, LNAI 5227, pp. 76-83, 2008.

[15] Q.X. Wu, T.M. McGinnity, L. P. Maguire, B.Glackin, and A. Belatreche, "Information Processing Functionality of Spiking Neurons for Image Feature Extraction", Seventh International Workshop on Information Processing in Cells and Tissue 2007, 29th 31 st August 2007, Jesus College, Oxford, UK

[16] W. Gerstner and W. Kistler, Spiking Neuron Models: Single Neurons, populations, Plasticity. Cambridge University Press, 2002.

[17] Q.X. Wu, T.M. McGinnity, L.P. Maguire, B. Glackin and A. Belatreche, "Learning Mechanism in Networks of Spiking Neurons",Studies in Computational Intelligence, LNCS, SpringerVerlag vol. 35, pp.171-197, 2007.

[18] P.V.C. Hough, "Method and means for recognizing complex patterns", U.S. Patent 2069 654, 18, 1962.

[19] R.O. Duda and P.E. Hart, "Use Hough transformation to detect lines and curves in pictures", Commun. Ass. Comput., Mach., vol. 15, pp.11-15, 1972. 